\title{
Marker-assisted backcrossing to improve seed oleic acid content in four elite and popular peanut (Arachis hypogaea L.) cultivars with high oil content
}

\author{
Bingyan Huang1), Feiyan Qi'), Ziqi Sun'), Lijuan Miao'), Zhongxin Zhang1), Hua Liu' ${ }^{2)}$, Yuanjin Fang'), \\ Wenzhao Dong'), Fengshou Tang'), Zheng Zheng') and Xinyou Zhang*1) \\ 1) Key Laboratory of Oil Crops in Huanghuaihai Plains, Industrial Crops Research Institute, Henan Academy of Agricultural Sciences, \\ 116 Huayuan Road, 450002, Zhengzhou, China \\ 2) Henan Provincial Key Laboratory for Oil Crops Improvement, 116 Huayuan Road, 450002, Zhengzhou, China
}

\begin{abstract}
High oleic acid composition is an important determinant of seed quality in peanut (Arachis hypogaea) in regard to its nutritional benefits for human health and prolonged shelf-life for peanut products. To improve the oleic acid content of popular peanut cultivars in China, four peanut cultivars of different market types were hybridized with high-oleic-acid donors and backcrossed for four generations as recurrent parents using fad2 marker-assisted backcross selection. Seed quality traits in advanced generations derived by selfing were assessed using near-infrared reflectance spectroscopy for detection of oleic acid and Kompetitive allele-specific PCR (KASP) screening of $f a d 2$ mutant markers. Twenty-four high-oleic-acid lines of $\mathrm{BC}_{4} \mathrm{~F}_{4}$ and $\mathrm{BC}_{4} \mathrm{~F}_{5}$ populations, with morphological features and agronomic traits similar to those of the recurrent parents, were obtained within 5 years. The genetic backgrounds of $\mathrm{BC}_{4} \mathrm{~F}_{5}$ lines were estimated using the KASP assay, which revealed the genetic background recovery rate was $79.49 \%-92.31 \%$. The superior lines raised are undergoing a multi-location test for cultivar registration and release. To our knowledge, this is the first application of single nucleotide polymorphism markers based on the high-throughput and cost-effective KASP assay for detection of $f a d 2$ mutations and genetic background evaluation in a peanut breeding program.
\end{abstract}

Key Words: peanut, Arachis hypogaea, high oleic acid, oil content, marker-assisted backcross selection, single nucleotide polymorphism, genetic background recovery.

\section{Introduction}

Peanut (Arachis hypogaea L.) is an economically important crop grown worldwide. The kernel is a valuable source of plant protein and edible oil. Peanut is grown in more than 100 countries, predominantly in Asia and Africa, and is a major contributor to farmers' net income and to essential nutritional requirements of humans (FAO 2016). The kernel oil content of peanut is about $50 \%$, and oleic and linoleic acid account for $80 \%$ of its fatty acid composition. High oleic acid content is a desirable trait associated with both the shelf life of processed peanut products and human health (Derbyshire 2014, Kris-Etherton et al. 1999, O'Keefe et al. 1993, Talcott et al. 2005a, 2005b, Vassiliou et al. 2009). Oleic acid content in conventional peanut cultivars is $48 \%-54 \%$, and in high-oleic-acid peanut cultivars the content may be as high as $80 \%$ (Norden et al. 1987).

Communicated by Sachiko Isobe

Received July 17, 2018. Accepted January 2, 2019.

First Published Online in J-STAGE on April 27, 2019.

*Corresponding author (e-mail: haasxinyou@163.com)
Norden et al. (1987) identified the first natural higholeic-acid peanut mutant F435, with seed oleic acid content as high as $80 \%$, in the Florida breeding program. Subsequently, the high-oleic-acid mutants M2-225 and C458 were generated by chemical mutagenesis in the United States (Patel et al. 2004). The discovery of high-oleic-acid mutants enhanced the isolation of, and functional research on, genes controlling oleic acid content in peanut. The fatty acid desaturase gene AhFAD2 is the critical gene that controls the conversion of oleic acid to linoleic acid, which determines the oleic and linoleic acid contents in peanut kernels (Jung et al. 2000a, 2000b). The tetraploid cultivated peanut genome contains two functional $A h F A D 2$ genes, named AhFAD2A $\left(\mathrm{Ol}_{1}\right)$ and AhFAD2B $\left(\mathrm{Ol}_{2}\right)$, which are located in the $\mathrm{A}$ and $\mathrm{B}$ genomes, respectively. Previous studies have provided evidence that AhFAD2 mutations reduce palmitic acid content while increasing oleic acid content in the peanut kernel (Pandey et al. 2012, Wang et al. 2015). Presently, only one mutation of $A h F a d 2 A\left(o l_{1}\right)$, which can result in high oleic acid content together with loss-of-function of $A h F a d 2 B\left(\mathrm{ol}_{2}\right)$, has been reported in peanut. The $o l_{1}$ mutant harbors a $\mathrm{G} \rightarrow \mathrm{A}$ substitution at the $448 \mathrm{bp}$ position of 
AhFad2A (López et al. 2000). Several types of mutations of $A h F a d 2 B$ based on different mutation origins have been reported. The natural mutation of AhFad2B in F435 is an A insertion at $442 \mathrm{bp}$, resulting in a frame shift and early termination of translation. The chemically induced mutations of $A h F a d 2 B$ in M2-225 and C458 are miniature inverted transportable element (MITE) insertions at $997 \mathrm{bp}$ and $665 \mathrm{bp}$, resulting in the loss of function of $A h F a d 2 B$ (Patel et al. 2004).

Molecular markers have been developed to assist in selection for the high-oleic-acid trait using different marker technologies for marker-assisted selection (MAS) in the last decade (Barkley et al. 2009, Chen et al. 2010, Chu et al. 2011). Near-infrared reflectance spectroscopy (NIR) provides low cost and rapid measurement of the oil content and fatty acid composition of intact seeds (Fox and Cruickshank 2005, Kandala et al. 2012, Tillman et al. 2006, Wang et al. 2013). However, thus far, few high-oleic-acid peanut cultivars have been released for commercial production, and the majority of these cultivars are derived from hybridization and pedigree selection. The genetic background of higholeic-acid donor parents is extremely narrow in the conventional peanut breeding germplasm, with the majority derived from small-seeded (less than $70 \mathrm{~g}$ hundred-seed weight; HSW) and late-maturing (more than $135 \mathrm{~d}$ ) cultivars (Baring et al. 2013, Burow et al. 2014, Gorbet and Knauft 1997, Simpson et al. 2003, 2006). Recently, much attention has been devoted to breeding high-oleic-acid peanut cultivars with expanded genetic backgrounds and diverse market types (Wang et al. 2015). On the Huang-HuaiHai Plain, which is the main peanut production region of China, peanut seeds are sown after wheat harvesting and the development of early-maturing cultivars (less than $120 \mathrm{~d}$ ) with large seeds (more than $80 \mathrm{~g} \mathrm{HSW}$ ) is a priority. Purposeful gene introgression is an effective means by which to introduce elite genes into existing germplasm or breeding populations, but it is a long-term and arduous process owing to the numerous backcrossing generations required. Alternatively, with the development of molecular marker technologies, marker-assisted backcrossing (MABC) shows promise for application in many crops. Under MABC, the selection of plants with heterozygous markers for the target trait to be crossed with the recurrent parent is more efficient and thus improves the accuracy and reduces the time and cost involved in the backcrossing process.

In China, about $55 \%$ of peanut kernels are processed for oil extraction (Wang 2018). As a consequence, kernel oil content is an extremely important trait and a breeding target for peanut breeding programs. 'Yuhua 15' (YH15), 'Yuanza 9102' (YZ9102), 'Yuhua 9327' (YH9327), and 'Yuhua 9326' (YH9326) are elite peanut cultivars with high oil content that were released in 2000, 2000, 2003, and 2005, respectively. YH15 and YH9326 are of the Virginia type with large seeds and YH9327 is a Virginia type with mediumsized seeds, whereas YZ9102 is a Spanish-type cultivar. These four cultivars are popular on account of their high oil content and high yield, and are still in cultivation as control cultivars in Henan province of China. However, the oleic acid contents of the elite cultivars are not high and need to be improved to meet the increasing market demand for high-oleic-acid peanut. To improve oleic acid content in the four cultivars while retaining the oil content and other favorable traits, the MABC breeding strategy was chosen instead of MAS because with the latter approach the higholeic-acid trait would segregate widely among the progeny during selfing generations and would require a greater number of generations and larger populations to obtain elite pure lines with high oleic acid contents. In the present study, four cross combinations were performed between the four elite conventional peanut cultivars with high kernel oil content (FAD2 genotype of $\mathrm{Ol}_{1} \mathrm{Ol}_{1} \mathrm{Ol}_{2} \mathrm{Ol}_{2}$ ) and high-oleic-acid donor cultivars (FAD2 genotype of $\mathrm{ol}_{1} \mathrm{ol}_{1} \mathrm{Ol}_{2} \mathrm{Ol}_{2}$ ) using MABC to improve oleic acid content in Chinese elite peanut cultivars. The $\mathrm{BC}_{4} \mathrm{~F}_{1}$ populations were obtained within three years from 2013 to 2015, and seeds of 56 pure lines were obtained from $\mathrm{BC}_{4} \mathrm{~F}_{3}$ to $\mathrm{BC}_{4} \mathrm{~F}_{5}$ that showed both high oleic acid and high oil contents. The newly developed lines corresponding to the recurrent parents YH15, YZ9102, YH9327, and YH9326 were named HOAPYH15, HOAPYZ9102, HOAPYH9327, and HOAPYH9326, respectively. Allelespecific PCR was used to assist in selection of the $\mathrm{Ol}_{1} \mathrm{Ol}_{1} \mathrm{Ol}_{2} \mathrm{Ol}_{2}$ genotype in $\mathrm{F}_{1}$ and $\mathrm{BC}_{n} \mathrm{~F}_{1}$ populations. The NIR phenotyping method in combination with Kompetitive allele-specific PCR (KASP) genotype screening were employed for $\_{ }_{0} l_{1_{-}} o l_{2}$ genotype selection in the selfing generations following the $\mathrm{BC}_{4} \mathrm{~F}_{1}$ generation. Phenotyping showed that backcrossing was effective in recovering favorable agronomic traits of the recurrent parents. In addition, single nucleotide polymorphism (SNP) markers between the two parental cultivars were selected to examine the genetic background recovery ratio in populations of different generations.

This study provides a rapid and low-cost approach to convert elite peanut cultivars from normal to high oleic acid contents. Compared with traditional backcrossing, the MABC approach saves time and costs for selfing of $F_{1}$ individuals to select the heterozygous genotypes harboring the recessive gene or marker for backcrossing. In addition, with the assistance of markers, the oleic acid traits can be selected in off-seasons, thus further saving on costs compared with traditional backcrossing in northern China, whereby usually no selection is conducted in the off-season and all progenies harvested in the off-season must be sown in the following growing season. We also tested and confirmed the oil and oleic acid contents by NIR and conducted a comprehensive comparison in $\mathrm{BC}_{n} \mathrm{~F}_{2: 3}$ generations of the different backcrosses. To our knowledge, this is the first report comparing trait recovery for oil content in different backcross generations and genetic recovery of recurrent parents by KASP screening using SNP markers located on all of the 20 chromosomes of cultivated peanut. The present results demonstrate that trait introgression using molecular markers 
to select on both the foreground (traits) and the background (genetic background) will soon be available for peanut with the development of genomic studies.

\section{Materials and Methods}

\section{Plant materials}

Four elite peanut cultivars were selected as female parents for crossing and recurrent backcrossing, namely 'Yuhua 15', 'Yuanza 9102', 'Yuhua 9326', and 'Yuhua 9327'. These cultivars have been popular in large growing areas in the main peanut production region in China in recent years ( $\mathrm{Yu}$ 2008). The HSW for each cultivar is $94.85 \pm 6.46,70.34$ $\pm 5.17,82.77 \pm 4.46$, and $93.37 \pm 4.68$, respectively. The male donor parents were either a released cultivar (Kainong176; KN176) or breeding lines (DF12 and KX016) with high oleic acid content and harboring mutations in both $A h F a d 2 A$ and $A h F a d 2 B$ genes of the F435 type $\left(\mathrm{ol}_{1} \mathrm{Ol}_{1} \mathrm{Ol}_{2} \mathrm{Ol}_{2}\right)$. Details of the agronomic traits and characteristics of the cultivars are listed in Table 1. KN176 and KX016 were provided by Jianzhong Gu from the Kaifeng Academy of Agricultural Research, Kaifeng, China, and the other cultivars were released by the Industrial Crops Research Institute of the Henan Academy of Agricultural Sciences.

All recurrent parents had higher oil content (more than $55 \%$ ) and early maturity (less than $120 \mathrm{~d}$ ) compared with those of the high-oleic-acid donor parents. YH15, YH9327, and YH9326 are large-seeded Virginia-type bunch cultivars with medium duration of maturation. YZ9102 is a Spanish type harboring bacterial wilt resistance and other desirable agronomic traits.

\section{Crossing combinations and selection strategy}

The first crossing season was initiated in the summer of 2013 for the four combinations designated as HAAS1 $($ YH15 $\times$ KN176), HAAS2 (YZ9102 $\times$ DF12), HAAS3 $($ YH9327 $\times$ KN176), and HAAS4 (YH9327 $\times$ KX01-6). In total, four cycles of backcrossing were performed with two seasons each year either in Zhengzhou (summer season) or in Sanya (winter season) from 2013 to 2014.

$\mathrm{F}_{1}$ individuals for backcrossing were genotyped by MAS and the selfing generations were selected for oleic acid content using NIR. We applied MAS to select the $\mathrm{Ol}_{1} \mathrm{Ol}_{1} \mathrm{Ol}_{2} \mathrm{Ol}_{2}$ genotypes in the $F_{1}$ generation for further backcrossing with recurrent parents, and harvested all plants in each $\mathrm{F}_{2}$ generation from the $\mathrm{BC}_{2}$ to the $\mathrm{BC}_{4}$ generations for further comparison of the effects of different backcross generations. In subsequent selfing generations from $\mathrm{F}_{3}$ individuals until the $\mathrm{F}_{6}$ or $\mathrm{BC}_{n} \mathrm{~F}_{5}$ generations, plants with superior agronomic traits and oleic acid content higher than $60 \%$ (as detected by NIR) were selected and retained for further analysis (Fig. 1).

\section{Allele-specific marker-assisted selection of fad2 mutation in $F_{1}$ generations}

Young leaves were collected from $F_{1}$ seedlings in each generation and DNA was extracted using the DNAsecure Plant Kit (TIANGEN BIOTECH CO., Beijing, China). DNA concentrations were checked using a NanoDrop spectrophotometer and adjusted to $50 \mathrm{ng} / \mu \mathrm{L}$ for use in PCR.

Genotyping of the $\mathrm{F}_{1}$ individuals with FAD2 markers was performed before further crossing activities, and confirmed hybrids for both genes with the $\mathrm{Ol}_{1} \mathrm{Ol}_{1} \mathrm{Ol}_{2} \mathrm{Ol}_{2}$ genotype were used either as pollen donors for further backcrossing $\left(\mathrm{BC}_{1} \mathrm{~F}_{1}\right.$, $\mathrm{BC}_{2} \mathrm{~F}_{1}$, and $\left.\mathrm{BC}_{3} \mathrm{~F}_{1}\right)$ to generate the backcross generations

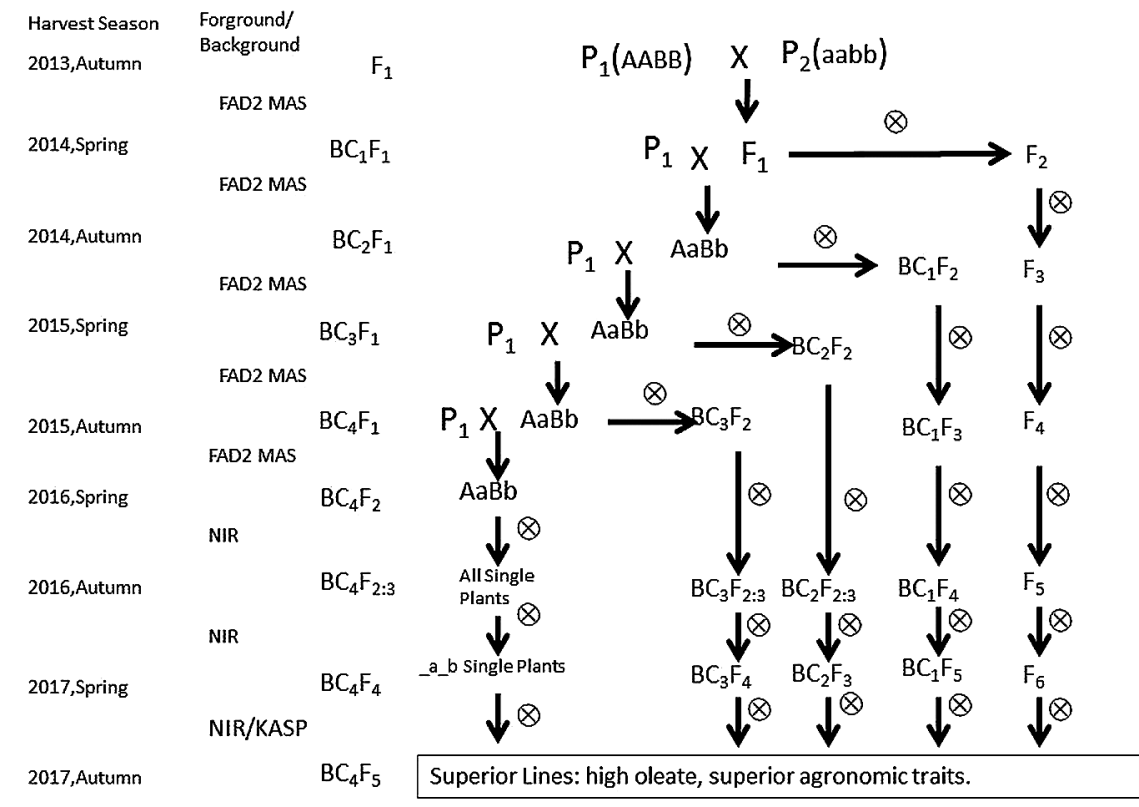

Fig. 1. Breeding process and selection strategy. FAD, fatty acid desaturase, MAS, marker-assisted selection; NIR, near-infrared reflectance spectroscopy. 
$\mathrm{BC}_{2} \mathrm{~F}_{1}, \mathrm{BC}_{3} \mathrm{~F}_{1}$, and $\mathrm{BC}_{4} \mathrm{~F}_{1}$, or selfed to obtain $\mathrm{F}_{2}$ generations for generation advancement. Individuals with the $\mathrm{Ol}_{1} \mathrm{Ol}_{1} \mathrm{Ol}_{2} \mathrm{Ol}_{2}, \mathrm{Ol}_{1} \mathrm{Ol}_{1} \mathrm{Ol}_{2} \mathrm{Ol}_{2}$, and $\mathrm{Ol}_{1} \mathrm{Ol}_{1} \mathrm{Ol}_{2} \mathrm{Ol}_{2}$ genotypes were discarded.

Four pairs of allele-specific primers, two pairs for amplification of the wild-type alleles $\left(\mathrm{Ol}_{1}\right.$ and $\left.O l_{2}\right)$ and two pairs to amplify mutant alleles $\left(\mathrm{ol}_{1}\right.$ and $\left.o l_{2}\right)$ of $A h F a d 2 A / B$, were applied for MAS using four PCR reactions as indicated in the patent (Zhang 2015): (a) FAD2A-F (aF19F; Patel et al. 2004): 5'-GATTACTGATTATTGACTT-3'; FAD2A-WT-R: 5'-GTTTTGGGACAAACACTTCACC-3' to amplify wildtype $\mathrm{AhFad} 2 \mathrm{~A}\left(\mathrm{Ol}_{1}\right)$; (b) FAD2A-F (aF19F): 5'-GATTACT GATTATTGACTT-3'; FAD2A-Mu-R: 5'-GTTTTGGGACA AACACTTAGAT-3' to amplify mutant genotype $A h F a d 2 a$ $\left(\mathrm{ol}_{l}\right)$; (c) FAD2B-F (bF19F; Patel et al. 2004): 5'-CAGAAC CATTAGCTTTG-3'; FAD2B-WT-R: 5'-GACAAACACTT CGTCGCGTTAG-3' to amplify wild-type $\operatorname{AhFadB}\left(\mathrm{Ol}_{2}\right)$; and (d) FAD2B-F (bF19F): 5'-CAGAACCATTAGCTTTG3'; FAD2B-Ins-R: 5'-GACAAACACTTCGTCGCGTTAT-3' to amplify mutant genotype $A h F a d 2 b\left(\mathrm{ol}_{2}\right)$.

The PCR reaction was carried out in a $10 \mu \mathrm{L}$ volume using 20 ng genomic DNA, $1 \mathrm{U}$ of LA Taq ${ }^{\circledR}$ DNA polymerase (TAKARA, Kusatsu, Japan), 1× PCR buffer, $0.4 \mathrm{mM}$ dNTPs, and $3 \mu \mathrm{M}$ each of forward and reverse primers. The PCR program included an initial denaturation step of $5 \mathrm{~min}$ at $94^{\circ} \mathrm{C}$, followed by 30 or 35 cycles at $94^{\circ} \mathrm{C}$ for $30 \mathrm{~s}$, $45.9^{\circ} \mathrm{C}$ for $30 \mathrm{~s}$, and $72^{\circ} \mathrm{C}$ for $45 \mathrm{~s}$, and final extension at $72^{\circ} \mathrm{C}$ for $10 \mathrm{~min}$. The number of cycles was 30 for reactions with FAD2A-F/FAD2A-WT-R and 35 for FAD2A-F/ FAD2A-Mu-R, FAD2B-F/FAD2B-WT-R, and FAD2B-F/ FAD2B-Ins-R. The amplified PCR products were separated by agarose gel electrophoresis and visualized under ultraviolet light using a VersaDoc system (Bio-Rad, Hercules, CA, USA).

KASP assay for fad2 mutation and genetic background recovery in advanced generations

Four generations of selfing were conducted following the $\mathrm{BC}_{4} \mathrm{~F}_{2}$ generation to obtain the $\mathrm{BC}_{4} \mathrm{~F}_{4}$ population from 2015 to 2016. The selection of single plants for generation advancement was assisted by NIR detection together with high-throughput KASP screening of FAD2 markers.

Individual plants from $\mathrm{BC}_{2} \mathrm{~F}_{4}, \mathrm{BC}_{3} \mathrm{~F}_{4}$, and $\mathrm{BC}_{4} \mathrm{~F}_{4}$ lines were collected in all four combinations in 2017. A total of 27 polymorphic KASP-based markers were selected from 144 validated SNP polymorphic loci discovered in a tunable genotyping-by-sequencing (tGBS) project with 320 germplasm. These markers were used to detect the recovery ratio of the genetic backgrounds of the four recurrent parents. The KASP reaction was in a $5 \mu \mathrm{L}$ PCR system on 384well plates, which included $2.5 \mu \mathrm{L}$ DNA template (10$20 \mathrm{ng}$ ), $2.43 \mu \mathrm{L}$ of $2 \times$ KASP Master Mix (LGC, Hoddesdon, UK), and $0.07 \mu \mathrm{L}$ KASP Assay mix. The assay mix was a mixture of two allele-specific forward primers and one reverse primer. Sequences of allele-specific and common primers used are listed in Supplemental Table 1. KASPar oligos were ordered from LGC. Amplification was performed on a PCR machine with 384 blocks using the following protocol: hot start at $94^{\circ} \mathrm{C}$ for $15 \mathrm{~min}$, followed by ten touchdown cycles $\left(94^{\circ} \mathrm{C}\right.$ for $20 \mathrm{~s}$; touchdown $61^{\circ} \mathrm{C}$, $-0.6^{\circ} \mathrm{C}$ per cycle, $60 \mathrm{~s}$ ), and then 26 cycles of amplification $\left(94^{\circ} \mathrm{C}\right.$ for $20 \mathrm{~s} ; 57^{\circ} \mathrm{C}$ for $60 \mathrm{~s}$ ) (Trick et al. 2012).

\section{Oil and oleic acid contents measurement}

Mature seeds harvested from $\mathrm{BC}_{n} \mathrm{~F}_{2}$ and $\mathrm{BC}_{4} \mathrm{~F}_{4}$ progenies were measured for oil content and oleic acid content by NIR. The NIR estimation for seed quality was conducted using a DA 7250 near infrared analyzer (Perten Instruments, Hägersten, Sweden). Five to ten seeds were analyzed for each sample with two replicates.

To confirm the accuracy of the NIR measurement, oil content and oleic acid content in peanut seeds from recent breeding materials were analyzed by gas chromatography (GC) at the Agricultural Product Quality Supervision and Inspection Center, Zhengzhou, of the Ministry of Agriculture of China. Sample pretreatment and esterification reactions were as follows. In total, a $0.5 \mathrm{~g}$ sample was tested in a $15 \mathrm{~mL}$ graduated test tube, with $5 \mathrm{~mL}$ of mixed ether and petroleum (1:1) added to dissolve the sample. Samples were vortexed for $1 \mathrm{~min}$ in a vortex generator and treated ultrasonically for $10 \mathrm{~min} ; 2 \mathrm{ml}$ of $5.4 \% \mathrm{KOH}$ methanol solution was added, then vortexed for $1 \mathrm{~min}$, followed by $20 \mathrm{~min}$ of ultrasonic treatment. Samples were left to react for $1 \mathrm{~h}$, and then $10 \mathrm{~mL}$ of $5 \% \mathrm{NaCl}$ aqueous solution was added. After static layering, the upper clear liquid was used for measurement. The instrument used for fatty acid composition analysis was an Agilent 6890N gas chromatograph (Agilent Technologies, Santa Clara, CA, USA). The sample size was $1 \mu \mathrm{L}$; shunt ratio $1: 100$; inlet temperature $250^{\circ} \mathrm{C}$; detector temperature $270^{\circ} \mathrm{C}$; and column temperature set for programmed increase with initial temperature $190^{\circ} \mathrm{C}$, increasing to $215^{\circ} \mathrm{C}$ at $3^{\circ} \mathrm{C} / \mathrm{min}$, and holding for $10 \mathrm{~min}$, then increasing to $230^{\circ} \mathrm{C}$ at $20^{\circ} \mathrm{C} / \mathrm{min}$, and holding for $5 \mathrm{~min}$.

\section{Statistical analysis}

Correlation analysis was conducted using Microsoft Excel. The $p$-values for pairwise comparisons were calculated using DPS 14.50 software (Tang and Zhang 2013).

\section{Results}

\section{Correlation of oleic acid and oil contents between $G C$ and NIR detection}

Near-infrared reflectance spectroscopy has the advantages of being rapid and non-destructive for detection of seed oil and oleic acid contents (Fox and Cruickshank 2005, Tillman et al. 2006, Wang et al. 2013). To verify the accuracy of the established NIR detection system for measurement of oil and oleic acid contents in our laboratory, 260 germplasm lines and 67 progeny generated in a high-oleic-acid peanut breeding program in recent years were selected for comparison of oil and oleic acid contents using NIR and GC. The 
coefficient of determination $\left(R^{2}\right)$ for oil content and oleic acid content was 0.6889 and 0.9604 , respectively. Compared with the corresponding genotyping results, the findings indicated that an individual plant with seed oleic acid content of more than $60 \%$ would possess at least one mutant allele for $f a d 2 a$ and $f a d 2 b$.

\section{Oil and oleic acid content frequency distribution in differ- ent backcross generations}

Oil content distribution in $B C_{2} F_{2}, B C_{3} F_{2}$, and $B C_{4} F_{2}$

In the backcrossing, the $\mathrm{BC}_{n-1} \mathrm{~F}_{1} A h F a d 2$ genotype used as the male parent was $\mathrm{Ol}_{1} \mathrm{Ol}_{1} \mathrm{Ol}_{2} \mathrm{Ol}_{2}$ and the four possible genotypes of $\mathrm{BC}_{n} \mathrm{~F}_{1}$ were $\mathrm{Ol}_{1} \mathrm{Ol}_{1} \mathrm{Ol}_{2} \mathrm{Ol}_{2}, \mathrm{Ol}_{1} \mathrm{Ol}_{1} \mathrm{Ol}_{2} \mathrm{Ol}_{2}$, $\mathrm{Ol}_{1} \mathrm{Ol}_{1} \mathrm{Ol}_{2} \mathrm{Ol}_{2}$, and $\mathrm{Ol}_{1} \mathrm{Ol}_{1} \mathrm{Ol}_{2} \mathrm{Ol}_{2}$. After removal of $\mathrm{BC}_{n} \mathrm{~F}_{1}$ individuals with genotypes of $\mathrm{Ol}_{1} \mathrm{Ol}_{1} \mathrm{Ol}_{2} \mathrm{Ol}_{2}, \mathrm{Ol}_{1} \mathrm{Ol}_{1} \mathrm{Ol}_{2} \mathrm{Ol}_{2}$, and $\mathrm{Ol}_{1} \mathrm{Ol}_{1} \mathrm{Ol}_{2} \mathrm{Ol}_{2}$, we harvested seeds from all plants of $\mathrm{BC}_{n} \mathrm{~F}_{2}$ lines derived from $\mathrm{BC}_{n} \mathrm{~F}_{1}$ lines with the genotype $\mathrm{Ol}_{1} \mathrm{Ol}_{1} \mathrm{Ol}_{2} \mathrm{Ol}_{2}$. The seeds were planted in 2016 to harvest the $\mathrm{BC}_{n} \mathrm{~F}_{2: 3}$ generation for comparison of oil and oleic acid contents among $\mathrm{BC}_{n} \mathrm{~F}_{2}$ lines (Table 1).

Oil content has been suggested to be controlled by several quantitative trait loci (QTLs), and has been considered a complex quality trait controlled by both genetic and environmental factors (Pandey et al. 2014). In the present study, the donor parents had oil contents of $53.43 \% \pm 0.71 \%$, $52.16 \% \pm 1.10 \%$, and $51.49 \% \pm 1.27 \%$ for KN176, DF 12 , and KX016, respectively, and the recurrent parents had oil contents of $56.70 \% \pm 0.34 \%, 57.40 \% \pm 1.07 \%, 55.26 \% \pm$ $0.45 \%$, and $56.67 \% \pm 0.75 \%$ for YH15, YZ9102, YH9327, and YH9326, respectively, indicating that there was considerable variation between the donor and recurrent parents. The pairwise comparisons of oil contents between YH15 and KN176, YZ9102 and DF12, YH9327 and KN176, and YH9326 and KX016 were all significant $(p<0.01)$. Hence, we used oil content as an indicator of genetic recovery, and analyzed the oil content in different generations to check whether this trait had regressed to the recurrent parents. In all four combinations, the frequency of high oil content attained its highest level in $\mathrm{BC}_{4}$ as expected (Fig. 2).

\section{Oleic acid content distribution in $B C_{n} F_{2}$}

The $\mathrm{F}_{2}$ population was derived from $\mathrm{F}_{1}$ individuals with the $\mathrm{Ol}_{1} \mathrm{Ol}_{1} \mathrm{Ol}_{2} \mathrm{Ol}_{2}$ genotype, thus the oleic acid content distribution in the $\mathrm{BC}_{2} \mathrm{~F}_{2}, \mathrm{BC}_{3} \mathrm{~F}_{2}$, and $\mathrm{BC}_{4} \mathrm{~F}_{2}$ generations for the four combinations were expected to show no differences between the $\mathrm{BC}$ generations. The frequency distribution of oleic acid content in the $\mathrm{BC}_{2} \mathrm{~F}_{2}, \mathrm{BC}_{3} \mathrm{~F}_{2}$, and $\mathrm{BC}_{4} \mathrm{~F}_{2}$ popula-

Table 1. Numbers of plants from $\mathrm{BC}_{n} \mathrm{~F}_{2}$ populations derived from $\mathrm{BC}_{n} \mathrm{~F}_{1}$ lines of the $\mathrm{Ol}_{1} \mathrm{Ol}_{1} \mathrm{Ol}_{2} \mathrm{Ol}_{2}$ genotype

\begin{tabular}{llllrc}
\hline \hline Combinations & $\begin{array}{c}\text { Recurrent } \\
\text { parents }\end{array}$ & $\begin{array}{l}\text { Donor } \\
\text { parents }\end{array}$ & $\mathrm{BC}_{2} \mathrm{~F}_{2: 3}$ & $\mathrm{BC}_{3} \mathrm{~F}_{2: 3}$ & $\mathrm{BC}_{4} \mathrm{~F}_{2: 3}$ \\
\hline HAAS1 & YH15 & KN176 & 114 & 101 & 55 \\
HAAS2 & YZ9102 & DF12 & 236 & 102 & 183 \\
HAAS3 & YH9327 & KN176 & 150 & 30 & 52 \\
HAAS4 & YH9326 & KX016 & 114 & 199 & 172 \\
\hline
\end{tabular}

tions indeed displayed a similar pattern of a long tail increasing towards high oleic acid content. The peak frequency was around $40 \%-45 \%$ and the frequency ranges of the long tails were from $60 \%$ to $80 \%$ (Fig. 3).

\section{KASP evaluation for background recovery}

In the $\mathrm{BC}_{4} \mathrm{~F}_{4}$ generation, we applied KASP screening to detect the background recovery percentage of the recurrent

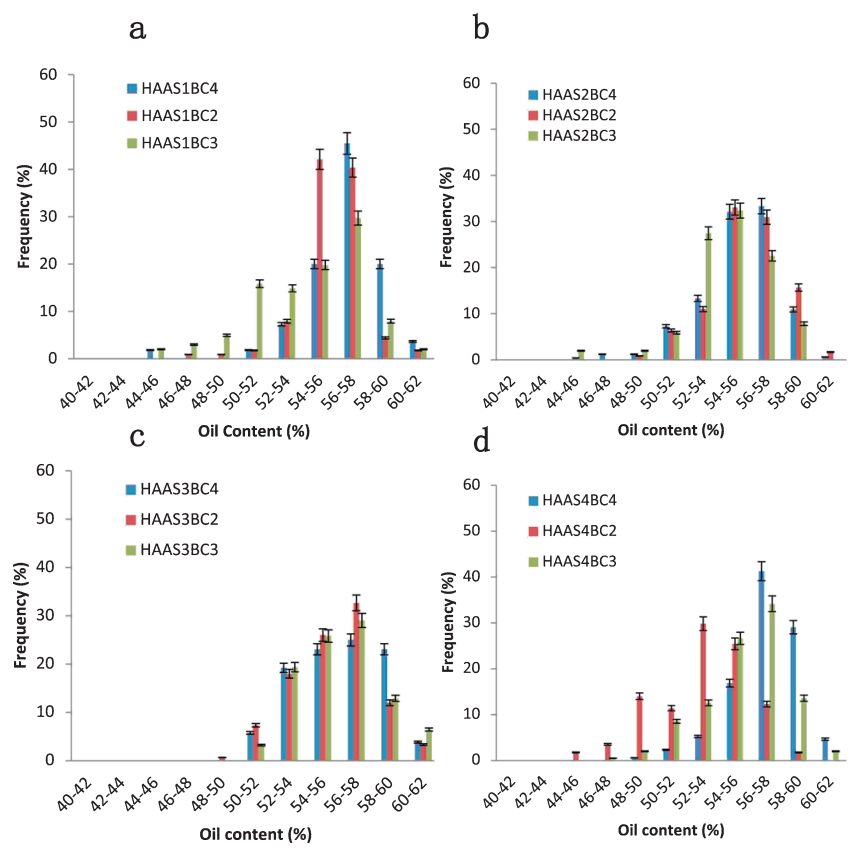

Fig. 2. Oil content distribution in $\mathrm{BC}_{n} \mathrm{~F}_{2}$. HAAS1, Yuhua $15 \times$ Kainong176; HAAS2, Yuanza9102 $\times$ DF12; HAAS3, Yuhua9327 $\times$ Kainong176; HAAS4, Yuhua9327 × KX01-6). a
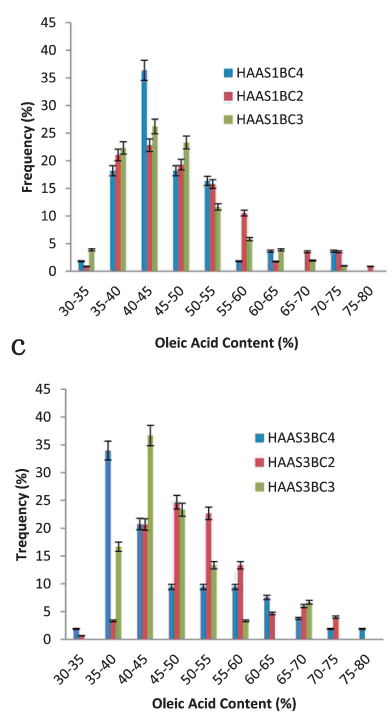

b
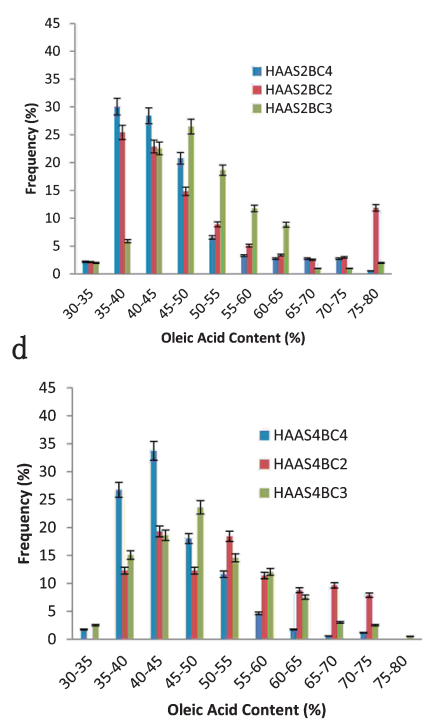

Fig. 3. Oleic acid content distribution in $\mathrm{BC}_{n} \mathrm{~F}_{2}$. HAAS1, Yuhua15 × Kainong176; HAAS2, Yuanza9102 $\times$ DF12; HAAS3, Yuhua9327 × Kainong176; HAAS4, Yuhua9327 × KX01-6). 
parent. From a total of 144 KASP markers, 27 were polymorphic between different pairs of parents and were used for single plant screening of the $\mathrm{BC}_{4} \mathrm{~F}_{4}$ population. The recovery percentage varied among the four combinations. While the theoretically expected recovery percentage on average was $96.88 \%$, the actual percentages ranged from $85.96 \%$ to $98.24 \%$ (Table 2, Supplemental Table 2). In the present study, HAAS1 and HAAS2 showed lower efficiency for genetic background recovery than HAAS3 and HAAS4. With regard to the background recovery of different backcross generations, even for HAAS3 and HAAS4 the percentages were less than theoretically expected in the $\mathrm{BC}_{2}$ and $\mathrm{BC}_{3}$ generations (Table 3, Supplemental Table 3).

To further validate the genetic background recovery of the selected lines, $123 \mathrm{BC}_{4} \mathrm{~F}_{5}$ lines from the four backcrossing combinations were screened using 88 polymorphic KASP markers. The results showed that the background recovery of the recurrent parent was $90.81 \%$ to $98.61 \%$ (Supplemental Table 4).

\section{Oleic acid and oil contents in superior lines}

Based on oil content, oleic acid content, and agronomic traits, 24 superior lines were selected for additional yield trials in multiple locations. The oleic acid and oil contents were similar to those of the high-oleic-acid donor parents and re-
Table 2. Recovery percentage of the genetic background of the recurrent parents in $\mathrm{BC}_{4} \mathrm{~F}_{4}$ lines

\begin{tabular}{lccc}
\hline \hline Generations & $\begin{array}{c}\text { No. of } \\
\text { datapoints }\end{array}$ & $\begin{array}{c}\text { No. of } \\
\text { positives* }\end{array}$ & $\begin{array}{c}\text { Recovery } \\
\text { percentage (\%) }\end{array}$ \\
\hline HAAS1BC ${ }_{4} \mathrm{~F}_{4}$ & 715 & 619 & 86.57 \\
HAAS2 $\mathrm{BC}_{4} \mathrm{~F}_{4}$ & 976 & 839 & 85.96 \\
HAAS3BC $\mathrm{F}_{4}$ & 360 & 336 & 93.33 \\
HAAS4BC $4 \mathrm{~F}_{4}$ & 624 & 613 & 98.24 \\
Average & & & 91.03 \\
\hline
\end{tabular}

* Having the same allele with recurrent parent.

Table 3. Recovery percentage of the genetic background of the recurrent parents in $\mathrm{BC}_{2} \mathrm{~F}_{4}$ and $\mathrm{BC}_{3} \mathrm{~F}_{4}$ lines

\begin{tabular}{lcccc}
\hline \hline Generations & $\begin{array}{c}\text { Expected } \\
\text { percentage (\%) }\end{array}$ & $\begin{array}{c}\text { No. of } \\
\text { datapoints }\end{array}$ & $\begin{array}{c}\text { No. of } \\
\text { positives* }\end{array}$ & $\begin{array}{c}\text { Recovery } \\
\text { percentage (\%) }\end{array}$ \\
\hline HAAS3BC $_{2}$ & 87.50 & 40 & 24 & 60.00 \\
HAAS4BC 2 & 87.50 & 196 & 151 & 77.04 \\
HAAS4BC & 93.75 & 238 & 215 & 90.34 \\
\hline
\end{tabular}

* Having the same allele with recurrent parent.

current parents (Figs. 4, 5). It was also observed that, coincident with the increase in oleic acid content, the palmitic acid content decreased by about $6 \%$ in the high-oleic-acid lines.

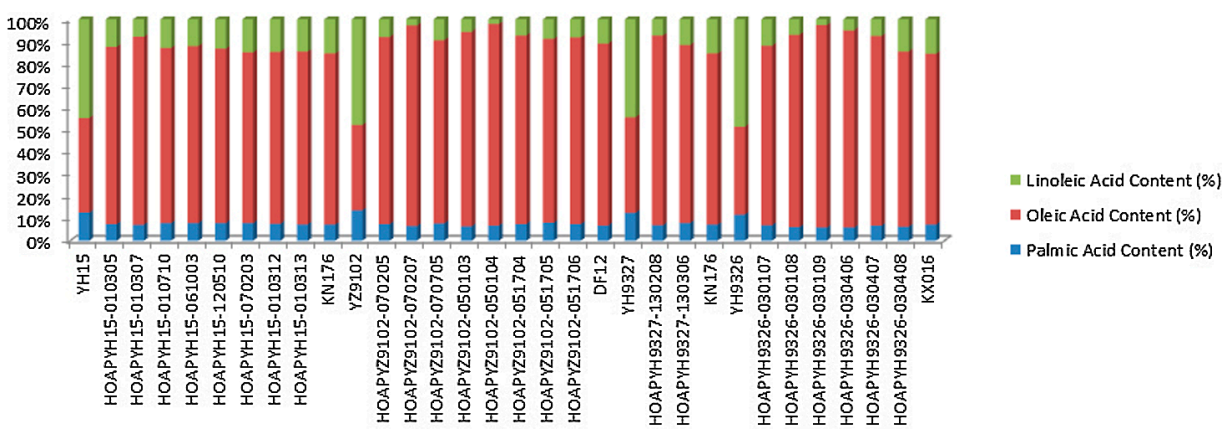

Fig. 4. Fatty acid content distribution in superior lines derived from the four backcross strategies. HOAP, high-oleic-acid peanut.

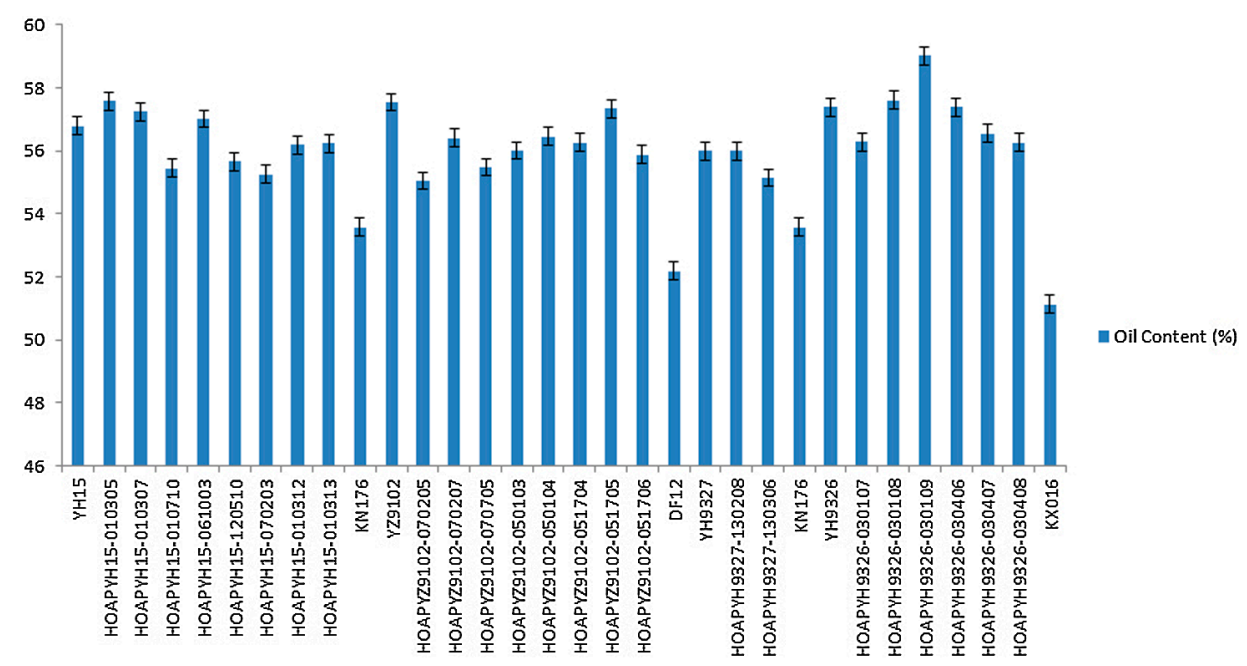

Fig. 5. Oil content distribution in superior lines derived from the four backcross strategies. HOAP, high-oleic-acid peanut. 


\section{Discussion}

Since the discovery of the high-oleic-acid peanut F435 in the Florida breeding program (Norden et al. 1987), many attempts have been made to introduce the $A h F a d 2$ mutation into elite cultivars by the conventional breeding methods of hybrid pedigree selection, or mutagenesis breeding by physical or chemical induction (Fang et al. 2012, Wang et al. 2011, 2015). The limited number of high-oleic-acid peanut germplasm lines available has resulted in the narrow genetic background of the released high-oleic-acid peanut cultivars, which usually have small pods with long duration of maturation (Nawade et al. 2016). Thus, the creation of a high-oleic-acid peanut with a broader genetic background is a matter of importance, and will benefit the breeding of high-oleic-acid peanut with large pods, early maturity, or resistance to various diseases. In previous studies, markerassisted breeding has been employed to generate peanut cultivars that carry nematode resistance, rust resistance, high oil content, and foliar disease resistance (Chu et al. 2011, Janila 2016a, Kolekar et al. 2017, Varshney et al. 2014). Compared with conventional breeding of high-oleicacid peanut, MABC and NIR may improve the accuracy and efficiency of high-oleic-acid trait selection. Chu et al. pyramided the high-oleic-acid trait with nematode resistance in the peanut cultivar 'Tifguard' using a MABC strategy and obtained $37 \mathrm{BC}_{3} \mathrm{~F}_{1}$ individuals heterozygous for a high oleic acid:linoleic acid ratio (Chu et al. 2011). In a recent report, Janila et al. (2016a) raised high-oleic-acid lines with either high oil content or low oil content in advanced generations derived from a $\mathrm{BC}_{1}$ population of three crosses between high-oleic-acid peanut and parents with different oil contents. In the present study, four elite peanut cultivars were subjected to successive MABC for four generations to generate a high-oleic-acid lines. Twenty-four superior high-oleic-acid lines were obtained that resembled the elite parents in morphological and agronomic traits, especially in oil content and early maturity.

\section{Recovery percentage in different backcross generations}

In crops for which extensive genomic and genetic information is available, genome selection and MAS are widely applied in plant breeding for background and foreground selection. For instance, in rice $65.55 \%-77.8 \%$ and $78.79 \%$ $95.5 \%$ recovery of the genetic background in the $\mathrm{BC}_{1} \mathrm{~F}_{1}$ and $\mathrm{BC}_{2} \mathrm{~F}_{1}$ generations from 13 double-heterozygous individuals has been achieved by MAS. In the $\mathrm{BC}_{2} \mathrm{~F}_{2}$ generation, the average recurrent parent genome recovery was $95.37 \%$ and that for the selected plant attained 96.3\% (Ahmed et al. 2015). In the case of marker-assisted introgression of bacterial blight and blast resistance into an elite maintainer line of rice, $\mathrm{MABC}$ to the $\mathrm{BC}_{3}$ generation yielded a single $\mathrm{BC}_{3} \mathrm{~F}_{1}$ plant that possessed the target genes with $\sim 94 \%$ recovery of the recurrent parent genome, whereas the best $\mathrm{BC}_{1} \mathrm{~F}_{1}$ plant that showed recovery of $73.41 \%$ was identified from nine double-heterozygous individuals (Balachiranjeevi et al.
2015). In pepper, one $\mathrm{BC}_{1} \mathrm{~F}_{1}$ individual showed $84 \%$ similarity to the recurrent parent from among 141 doubleheterozygous individuals, and seven $\mathrm{BC}_{2} \mathrm{~F}_{1}$ plants showed more than $96 \%$ recovery of the recurrent parent genotype from among 89 double-heterozygous individuals (Jeong et al. 2015).

In the present study, we conducted background phenotyping in advanced generations to evaluate and select lines with the desired agronomic traits. By comparing $\mathrm{BC}_{4} \mathrm{~F}_{2}$, $\mathrm{BC}_{3} \mathrm{~F}_{2}$, and $\mathrm{BC}_{2} \mathrm{~F}_{2}$ lines, we showed that the proportion of oil content is higher in $\mathrm{BC}_{4} \mathrm{~F}_{2}$ than in $\mathrm{BC}_{3} \mathrm{~F}_{2}$ and $\mathrm{BC}_{2} \mathrm{~F}_{2}$ lines. A greater number of plants with high oil content (the trait derived from the recurrent parents) and higher mean oil content were observed in $\mathrm{BC}_{4} \mathrm{~F}_{2}$ than in $\mathrm{BC}_{3} \mathrm{~F}_{2}$ lines. However, substantial numbers of plants with high oil content were obtained in the $\mathrm{BC}_{3} \mathrm{~F}_{2}$ population, indicating that three backcrosses are sufficient to recover this trait. For introgression of mutant FAD2 alleles into recipient parents to obtain lines with high or low oil content and with high oleic acid content, Janila et al. (2016a) only employed one backcrossing generation followed by five generations of selfing, and successfully obtained new lines with the target traits.

In the present experiment, genotyping and phenotyping of background traits, such as oil content, were not employed to select desirable plants as pollen donors because the $\mathrm{F}_{1}$ populations were limited in size. Therefore, a single plant was randomly selected in each generation for successive backcrossing and the percentage of oil content in $\mathrm{BC}_{3} \mathrm{~F}_{2}$ lines may not be higher than that of $\mathrm{BC}_{2} \mathrm{~F}_{2}$ lines as expected. If the $F_{1}$ population is large enough to allow phenotyping for the background, or if marker-assisted background selection or genomic background selection becomes available in the future, the chance of high recovery ratios may be greatly increased even in the $\mathrm{BC}_{2} \mathrm{~F}_{2}$ or $\mathrm{BC}_{1} \mathrm{~F}_{2}$ generation. Three or four backcrosses are preferable and necessary in the event that $\mathrm{F}_{1}$ seeds are limited or the desired traits are controlled by many minor genes or QTLs.

\section{Genetic background selection in MABC}

With regard to MABC, the ideal strategy is to conduct foreground selection (of target genes from the donor parent) using tightly linked or functional markers, and background selection using parental polymorphic markers evenly distributed throughout the genome. In rice, maize, and other crops for which complete genomes have been assembled, background selection in MABC breeding programs is successfully deployed. Balachiranjeevi et al. (2015) applied 79 parental polymorphic simple sequence repeat (SSR) markers spread across the 12 chromosomes of rice for background selection in the $\mathrm{BC}_{2} \mathrm{~F}_{4}$ generation in the markerassisted introgression of bacterial blight and blast resistance into an elite maintainer line of rice. Jeong et al. (2015) used 204 polymorphic SNP markers evenly distributed throughout the pepper genome for background selection in MABC for development of pepper cultivars containing high contents of capsinoids. In a backcrossing breeding program 
for introgression of the $S u b 1$ gene into rice, a set of 88 polymorphic SSR markers were applied for recurrent parent genome recovery (Ahmed et al. 2015). However, the tetraploid cultivated peanut genome has not been fully assembled and re-sequencing projects by different research groups are ongoing, hampering the discovery of polymorphic sites. Although several studies have developed SSR-based markers for genetic mapping of disease resistance, drought tolerance, pod characteristics, and other important traits in peanut, SSR markers have been poorly applied in MAS procedures. This is mainly owing to the low genetic variance within cultivated peanut. Gautami et al. (2012) integrated genetic maps derived from three recombinant inbred line populations to acquire an integrated map with only 293 SSR markers. Furthermore, it is difficult to select evenly distributed markers for background selection with SSR markers, and some genetic mapping studies have developed fewer than 20 linkage groups, indicating the challenge of compiling a marker set to cover the whole genome. Previously, only two successful cases of MABC in peanut have been reported (Chu et al. 2011, Janila et al. 2016b), but neither study applied background selection.

Peanut has a large genome of approximately $2.8 \mathrm{~Gb}$ with $97 \%$ similarity between the A and B genomes, and containing more than $67 \%$ repeat sequences (Bertioli et al. 2016, Clevenger et al. 2017). This has impeded the development of high-throughput markers such as SNPs for use in breeding programs. With the release of the tetraploid cultivated peanut genome sequence and QTL mapping of important agronomic traits, more markers were expected to be used in MAS breeding of peanut (Chen et al. 2016, Luo et al. 2017a, 2017b, Pandey et al. 2012, Varshney 2016). In the present study, we selected 144 SNP loci from a tGBS project (Zheng et al., unpublished) and genotyped the peanut cultivars used in this study by means of a KASP assay. In total, 27 of the SNP markers showed polymorphism between the recurrent and donor parents. These SNP markers were used to estimate the genetic recovery ratio in different $\mathrm{BC}$ generations. Generally, when comparing the recovery rates in different generations of backcrossing, in a population size equivalent to those used in conventional breeding, three or four generations of backcrossing would be necessary to convert a normal-oleic-acid peanut cultivar to a high-oleic-acid peanut while retaining desirable multiple quantitative traits. This is consistent with results in rapeseed and maize when the transfer of the target traits involved distant hybridization or complex traits (Môro et al. 2017, Yu et al. 2017). The genetic background analysis in this study revealed that, on average, $68.52 \%, 90.34 \%$, and $91.03 \%$ of the 27 polymorphic alleles in the $\mathrm{BC}_{2}, \mathrm{BC}_{3}$, and $\mathrm{BC}_{4}$ generations, respectively, were identical to those of the recurrent parent.

Theoretically, the recovery rate is determined by four factors. The first is genome size: the smaller the genome, the more rapid the recovery. The second is the complexity of the traits: the fewer genes or QTLs that control the traits, the easier they are to recover. The third is the number of traits one aims to recover: the fewer, the easier. In a given species, the pivotal factor should be the population size in each backcross generation for full exchange and recombination among or within chromosome segments. The fourth factor is the trait difference between the two parents: the smaller, the easier. In the present study, for the HAAS1 combination, the two parents YH15 and KN176 show a small difference in oil content in contrast to HAAS2, for which YZ9102 and DF12 show a larger difference in oil content. Hence, in the same backcross generation, oil content recovery rates were higher in HAAS1 than in HAAS2.

Near-infrared reflectance spectroscopy has been used in the detection of seed quality characteristics in several crops. In the current study, application of NIR for selection of oleic acid content also benefits the high-oleic-acid peanut breeding program by detecting the content in early generations without destructive sampling of the seeds, thus reducing costs and enhancing the breeding process. It has been a routine procedure for high-oleic-acid peanut breeding to combine the NIR technique with KASP screening of $f a d 2$ mutations in our breeding program. To our knowledge, this is the first report of application of KASP assays for background evaluation in a peanut breeding program. We have widely utilized these assays in our genotyping laboratories to facilitate the screening of the genetic background of parental lines as well as for evaluation of hybridization progenies. The present study is the third report of applying fad 2 functional SNP markers to assist backcrossing selection in high-oleic-acid peanut breeding. However, it is the first application of high-throughput KASP assay of $f a d 2$ mutations, whereas previous studies applied allele-specific PCR and cleaved amplified polymorphic sequences (CAPS) markers. The current study provides proof of concept for a strategy for improving the oleic acid content of elite peanut cultivars and generating high-oleic-acid peanut germplasm with desired target traits in an accurate and efficient manner. The derived superior lines are currently undergoing a multilocation test for cultivar registration and release. The near-isogenic lines raised in the $\mathrm{BC}_{4}$ generation may also serve as a genetic resource for future research.

\section{Acknowledgments}

The work presented in this study was funded by Mars Chocolate North America, USA. This work has been undertaken as part of the breeding work of CARS (China Agricultural Research System, ARS-13), Henan Agriculture Research System (S2012-5), and Henan Province Open Cooperation Project of Science and Technology (No. 172106000007). We thank staff at Liwen Bianji, Edanz Editing China (www. liwenbianji.cn/ac), for critically reviewing a draft of this manuscript. 


\section{Literature Cited}

Ahmed,F., M.Y.Rafil, M.R. Ismail, A.S.Juraimi, H.A.Rahim, F.A. Tanweer and M.A.Latif (2015) Recurrent parent genome recovery in different populations with the introgression of $S u b 1$ gene from a cross between MR219 and Swarna-Sub1. Euphytica 207: 605-618.

Balachiranjeevi,C.H., N.S. Bhaskar, V.Abhilash, S.Akanksha, B.C. Viraktamath, M.S. Madhav, A.S.Hariprasad, G.S.Laha, M.S. Prasad, S.M. Balachandran et al. (2015) Marker-assisted introgression of bacterial blight and blast resistance into DRR17B, an elite, fine-grain type maintainer line of rice. Mol. Breed. 35: 151.

Baring, M.R., C.E.Simpson, M.D. Burow, J.M.Cason and J.L.Ayers (2013) Registration of 'Tamrun OL11' peanut. J. Plant Regist. 7: 154-158.

Barkley, N.A., K.D.C.Chamberlin, M.L.Wang and R.N.Pittman (2009) Development of a real-time PCR genotyping assay to identify high oleic acid peanuts (Arachis hypogaea L.). Mol. Breed. 25: 541-548.

Bertioli, D.J., S.B.Cannon, L.Froenicke, G.Huang, A.D.Farmer, E.K.S.Cannon, X. Liu, D. Gao, J.Clevenger, S. Dash et al. (2016) The genome sequences of Arachis duranensis and Arachis ipaensis, the diploid ancestors of cultivated peanut. Nat. Genet. 48: 438-446.

Burow, M.D., M.R. Baring, J.L.Ayers, A.M.Schubert, Y.López and C.E. Simpson (2014) Registration of 'Tamrun OL12' peanut. J. Plant Regist. 8: 117-121.

Chen, W., Y.Jiao, L.Cheng, L. Huang, B.Liao, M.Tang, X. Ren, X.Zhou, Y.Chen and H. Jiang (2016) Quantitative trait locus analysis for pod- and kernel-related traits in the cultivated peanut (Arachis hypogaea L.). BMC Genet. 17: 25.

Chen,Z., M.L. Wang, N.A. Barkley and R.N.Pittman (2010) A simple allele-specific PCR assay for detecting FAD2 alleles in both A and $\mathrm{B}$ genomes of the cultivated peanut for high-oleate trait selection. Plant Mol. Biol. Rep. 28: 542-548.

Chu, Y., C.L.Wu, C.C.Holbrook, B.L. Tillman, G.Person and P.OziasAkins (2011) Marker-assisted selection to pyramid nematode resistance and the high oleic trait in peanut. Plant Genome 4: 110-117.

Clevenger, J., Y.Chu, C.Chavarro, G.Agarwal, D.J.Bertioli, S.C.M. Leal-Bertioli, M.K. Pandey, J.Vaughn, B.Abernathy, N.A. Barkley et al. (2017) Genome-wide SNP genotyping resolves signatures of selection and tetrasomic recombination in peanut. Mol. Plant 10: 309-322.

Derbyshire,E.J. (2014) A review of the nutritional composition, organoleptic characteristics and biological effects of the high oleic peanut. Int. J. Food. Sci. Nutr. 65: 781-790.

Fang, C.Q., C.T. Wang, P.W. Wang, Y.Y. Tang, X.Z.Wang, F.G. Cui and S.L. Yu (2012) Identification of a novel mutation in FAD2B from a peanut EMS mutant with elevated oleate content. J. Oleo. Sci. 61: $143-148$

FAO Stat. (2016) http://faostat.fao.org/ (Access December 10, 2017)

Fox, G. and A. Cruickshank (2005) Near infrared reflectance as a rapid and inexpensive surrogate measure for fatty acid composition and oil content of peanut (Arachis hypogaea L.). J. Near Infrared Spectrosc. 13: 287-291.

Gautami,B., D.Foncéka, M.K.Pandey, M.C.Moretzsohn, V.Sujay, H. Qin, Y. Hong, I. Faye, X. Chen, A. BhanuPrakash et al. (2012) An international reference consensus genetic map with 897 marker loci based on 11 mapping populations for tetraploid groundnut (Arachis hypogaea L.) . PLoS ONE 7: e41213.

Gorbet, W. and A. Knauft (1997) Registration of 'SunOleic 95R' peanut. Crop Sci. 37: 1392
Huang, Qi, Sun, Miao, Zhang, Liu, Fang, Dong, Tang, Zheng et al.

Janila, P., M.K. Pandey, Y. Shasidhar, M.T. Variath, M. Sriswathi, P. Khera, S.S. Manohar, P. Nagesh, M.K. Vishwakarma, G.P. Mishra et al. (2016a) Molecular breeding for introgression of fatty acid desaturase mutant alleles (ahFAD2A and $a h F A D 2 B$ ) enhances oil quality in high and low oil containing peanut genotypes. Plant Sci. 242: 203-213.

Janila, P., M.T. Variath, M.K. Pandey, H. Desmae, B.N. Motagi, P. Okori, S.S. Manohar, A.L. Rathnakumar, T. Radhakrishnan, B. Liao et al. (2016b) Genomic tools in groundnut breeding brogram: status and perspectives. Front. Plant Sci. 7: 289.

Jeong, H.S., S. Jang, K. Han, J.K. Kwon and B.C. Kang (2015) Markerassisted backcross breeding for development of pepper varieties (Capsicum annuum) containing capsinoids. Mol. Breed. 35: 226.

Jung, S., D. Swift, E. Sengoku, M. Patel, F. Teulé, G. Powell, K. Moore and A.Abbott (2000a) The high oleate trait in the cultivated peanut [Arachis hypogaea L.]. I. Isolation and characterization of two genes encoding microsomal oleoyl-PC desaturases. Mol. Gen. Genet. 263: 796-805.

Jung, S., G. Powell, K. Moore and A.Abbott (2000b) The high oleate trait in the cultivated peanut [Arachis hypogaea L.]. II. Molecular basis and genetics of the trait. Mol. Gen. Genet. 263: 806-811.

Kandala, C.V., J.Sundaram and N. Puppala (2012) Analysis of moisture content, total oil and fatty acid composition by NIR reflectance spectroscopy: A Review. Mukhopadhyay, S.C. (ed.) Smart Sensing Technology for Agriculture and Environmental Monitoring (LNEE vol. 146), Springer, pp. 59-80.

Kolekar, R.M., M. Sukruth, K. Shirasawa， H.L.Nadaf， B.N. Motagi, S. Lingaraju, P.V.Patil and R.S. Bhat (2017) Marker-assisted backcrossing to develop foliar disease-resistant genotypes in TMV 2 variety of peanut (Arachis hypogaea L.). Plant Breed. 136: 948953.

Kris-Etherton, P.M., T.A. Pearson, Y.Wan, R.L. Hargrove, K. Moriarty, V.Fishell and T.D.Etherton (1999) High-monounsaturated fatty acid diets lower both plasma cholesterol and triacylglycerol concentrations. Am. J. Clin. Nutr. 70: 1009-1015.

López,Y., H.L. Nadaf, O.D. Smith, J.P.Connell, A.S. Reddy and A.K. Fritz (2000) Isolation and characterization of the $\Delta 12$-fatty acid desaturase in peanut (Arachis hypogaea L.) and search for polymorphisms for the high oleate trait in Spanish market-type lines. Theor. Appl. Genet. 101: 1131-1138.

Luo, H., X.Ren, Z.Li，Z.Xu，X.Li，L.Huang, X.Zhou, Y.Chen, W. Chen, Y. Lei et al. (2017a) Co-localization of major quantitative trait loci for pod size and weight to a $3.7 \mathrm{cM}$ interval on chromosome A05 in cultivated peanut (Arachis hypogaea L.). BMC Genomics 18: 58

Luo, H., Z.Xu, Z.Li, X.Li, J.Lv, X.Ren, L.Huang, X.Zhou, Y.Chen, J. Yu et al. (2017b) Development of SSR markers and identification of major quantitative trait loci controlling shelling percentage in cultivated peanut (Arachis hypogaea L.). Theor. Appl. Genet. 130: $1635-1648$.

Môro, G.V., M.F. Santos and C.L.J. Souza (2017) Use of genomic and phenotypic selection in lines for prediction of testcrosses in maize II: grain yield and plant traits. Euphytica 213: 128 .

Nawade,B., T.C.Bosamia, R. Thankappan, A.L. Rathnakumar, A. Kumar, J.R. Dobaria, R. Kundu and G.P.Mishra (2016) Insights into the indian peanut genotypes for ahFAD2 gene polymorphism regulating its oleic and linoleic acid fluxes. Front. Plant Sci. 7: 1271.

Norden,A.J., D.W. Gorbet, D.A. Knauft and C.T.Young (1987) Variability in oil quality among peanut genotypes in the Florida breeding program. Peanut Sci.14: 7-11. 
O'Keefe, S.F., V.A. Wiley and D.A. Knauft (1993) Comparison of oxidative stability of high- and normal-oleic peanut oils. J. Am. Oil Chem. Soc. 70: 489-492.

Pandey, M.K., E.Monyo, P.Ozias-Akins, X.Liang, P.Guimarães, S.N.Nigam, H.D. Upadhyaya, P.Janila, X.Zhang, B.Guo et al. (2012) Advances in Arachis genomics for peanut improvement. Biotechnol. Adv. 30: 639-651.

Pandey, M.K., M.L.Wang, L. Qiao, S.Feng, P.Khera, H.Wang, B. Tonnis, N.A. Barkley, J. Wang, C.C. Holbrook et al. (2014) Identification of QTLs associated with oil content and mapping FAD2 genes and their relative contribution to oil quality in peanut (Arachis hypogaea L.). BMC Genet. 15: 133.

Patel,M., S.Jung, K. Moore, G.Powell, C.Ainsworth and A.Abbott (2004) High-oleate peanut mutants result from a MITE insertion into the FAD2 gene. Theor. Appl. Genet. 108: 1492-1502.

Simpson, C.E., M.R. Baring, A.M. Schubert, H.A. Melouk, M.C. Black, Y.Lopez and K.A.Keim (2003) Registration of 'Tamrun OL01' peanut. Crop Sci. 43: 2298.

Simpson, C.E., M.R. Baring, A.M. Schubert, M.C. Black, H.A. Melouk and Y.Lopez (2006) Registration of 'Tamrun OL 02' peanut. Crop Sci. 46: 1813 .

Talcott, S.T., C.E. Duncan, D.D. Pozo-Insfran and D.W. Gorbet (2005a) Polyphenolic and antioxidant changes during storage of normal, mid, and high oleic acid peanuts. Food Chem. 89: 77-84.

Talcott, S.T., S.Passeretti, C.E.Duncan and D.W.Gorbet (2005b) Polyphenolic content and sensory properties of normal and high oleic acid peanuts. Food Chem. 90: 379-388.

Tang, Q.Y. and C.X. Zhang (2013) Data Processing System (DPS) software with experimental design, statistical analysis and data mining developed for use in entomological research. Insect Sci. 20: 254 260.

Tillman, B.L., D.W. Gorbet and G. Person (2006) Predicting oleic and linoleic acid content of single peanut seeds using near-infrared reflectance spectroscopy. Crop Sci. 46: 2121-2126.

Trick, M., N.M.Adamski, S.G. Mugford, C.C.Jiang, M.Febrer and C. Uauy (2012) Combining SNP discovery from next-generation sequencing data with bulked segregant analysis (BSA) to fine-map genes in polyploid wheat. BMC Plant Biol. 12: 14.

Varshney, R.K., M.K. Pandey, P. Janila, S.N. Nigam, H. Sudini, M.V.C.
Gowda, M. Sriswathi, T. Radhakrishnan, S.S. Manohar and P. Nagesh (2014) Marker-assisted introgression of a QTL region to improve rust resistance in three elite and popular varieties of peanut (Arachis hypogaea L.). Theor. Appl. Genet. 127: 1771-1781.

Varshney, R.K. (2016) Exciting journey of 10 years from genomes to fields and markets: Some success stories of genomics-assisted breeding in chickpea, pigeonpea and groundnut. Plant Sci. 242: 98-107.

Vassiliou, E.K., A. Gonzalez, C. Garcia, J.H.Tadros, G. Chakraborty and J.H. Toney (2009) Oleic acid and peanut oil high in oleic acid reverse the inhibitory effect of insulin production of the inflammatory cytokine TNF- $\alpha$ both in vitro and in vivo systems. Lipids Health Dis. 8: 25.

Wang, C.T., X.Z.Wang, Y.Y.Tang, Q.Wu, J.Z.Xu, D.Q.Hu and B.Qu (2013) Predicting main fatty acids, oil and protein content in intact single seeds of groundnut by near infrared reflectance spectroscopy. Adv. Mat. Res. 860-863: 490-496.

Wang, M.L., N.A.Barkley, Z.Chen and R.N.Pittman (2011) FAD2 gene mutations significantly alter fatty acid profiles in cultivated peanuts (Arachis hypogaea). Biochem. Genet. 49: 748-759.

Wang, M.L., B.Tonnis, C.Y.Q.An, D.Pinnow, V.Tishchenko and G.A.Pederson (2015) Newly identified natural high-oleate mutant from Arachis hypogaea L. subsp. hypogaea. Mol. Breed. 35: 186.

Wang, Q. (2018) Peanut processing characteristics and quality evaluation. Springer Nature Singapore Pte Lt. P1-5.

Wang, X.Z., Y.Y.Tang, Q.Wu, Q.X.Sun, Y.Y.Wang, D.Q.Hu and C.T.Wang (2015) Characterization of high-oleic peanut natural mutants derived from an intersectional cross. Grasas y Aceites 66: e091.

Yu, H., Z. Li, L. Yang, Y.Liu, M.Zhuang, L.Zhang, H.Lv, Z. Li, F. Han, X. Liu et al. (2017) Morphological and molecular characterization of the second backcross progenies of Ogu-CMS Chinese kale and rapeseed. Euphytica 213: 55.

Yu, S. (2008) Chinese peanut variety and pedigree. Shanghai, Shanghai Science and Technology Press, pp. 322-345.

Zhang, X., F. Qi, B.Huang, H.Liu, S.Han, W. Dong, F. Tang and J. Xu (2015) Molecular marker of high oleate peanut and its application in assistant selection in backcross breeding. Chinese Patent, ZL 201310434389.6 